PROCEEDINGS OF THE

AMERICAN MATHEMATICAL SOCIETY

Volume 127, Number 4, April 1999, Pages 1075-1077

S 0002-9939(99)04964-3

\title{
THE GROWTH THEOREM OF CONVEX MAPPINGS ON THE UNIT BALL IN $\mathbb{C}^{n}$
}

\author{
HIDETAKA HAMADA
}

(Communicated by Steven R. Bell)

\begin{abstract}
Let $\|\cdot\|$ be an arbitrary norm on $\mathbb{C}^{n}$. Let $f$ be a normalized biholomorphic convex mapping on the unit ball in $\mathbb{C}^{n}$ with respect to the norm $\|\cdot\|$. We will give an upper bound of the growth of $f$.
\end{abstract}

Let $\Omega$ be a domain in $\mathbb{C}^{n}$ which contains the origin in $\mathbb{C}^{n}$. A holomorphic mapping $f$ from $\Omega$ to $\mathbb{C}^{n}$ is said to be normalized, if $f(0)=0$ and the Jacobian matrix at the origin $D f(0)$ is the identity matrix. Let $\mathbb{B}^{n}$ denote the Euclildean unit ball in $\mathbb{C}^{n}$. Let $f(z)$ be a normalized biholomorphic convex mapping on $\mathbb{B}^{n}$. Then FitzGerald and Thomas [2], Liu [6] and Suffridge [7] independently used different methods to prove the following growth theorem.

$$
\frac{|z|}{1+|z|} \leq|f(z)| \leq \frac{|z|}{1-|z|}
$$

where $|\cdot|$ denotes the Euclidean norm. Let

$$
B_{p}=\left\{z \in \mathbb{C}^{n} ;\|z\|_{p}=\left(\sum_{i=1}^{n}\left|z_{i}\right|^{p}\right)^{1 / p}<1\right\}
$$

for $p \geq 1$. Gong and Liu [4] gave the following upper bound of the growth of normalized biholomorphic convex mappings on $B_{p}$.

$$
\|f(z)\|_{p} \leq \frac{\|z\|_{p}}{1-\|z\|_{p}} .
$$

They also obtained an upper bound of the growth of normalized biholomorphic convex mappings on the convex complex ellipsoid

$$
D\left(p_{1}, \ldots, p_{n}\right)=\left\{z \in \mathbb{C}^{n} ;\left|z_{1}\right|^{p_{1}}+\cdots+\left|z_{n}\right|^{p_{n}}<1\right\}
$$

with $p_{1}, \ldots, p_{n} \geq 1$.

Let $\|\cdot\|$ be an arbitrary norm on $\mathbb{C}^{n}$ and let $\mathbb{B}$ denote the unit ball in $\mathbb{C}^{n}$ with respect to the norm $\|\cdot\|$. Using the idea of FitzGerald and Thomas [2], we obtain the following theorem.

Received by the editors June 3, 1997 and, in revised form, July 16, 1997.

1991 Mathematics Subject Classification. Primary 32H02; Secondary 30C45.

(C)1999 American Mathematical Society 
Theorem. Let $f(z)$ be a normalized biholomorphic convex mapping from $\mathbb{B}$ to $\mathbb{C}^{n}$. Then

$$
\|f(z)\| \leq \frac{\|z\|}{1-\|z\|}
$$

Proof. Let $\Delta$ be the unit disc in $\mathbb{C}$. For any fixed $w \in \partial \mathbb{B}$ and $\zeta \in \Delta$, let

$$
f(\zeta w)=\zeta w+\sum_{i=2}^{\infty} d_{i} \zeta^{i}
$$

Since $f$ is a holomorphic mapping into $\mathbb{C}^{n}, d_{i} \in \mathbb{C}^{n}$. Let $m \geq 2, m \in \mathbb{Z}$ be fixed. Let $\varepsilon=\exp (2 \pi i / m)$. Then

$$
\sum_{k=0}^{m-1} f\left(\zeta^{1 / m} \varepsilon^{k} w\right)=m \sum_{i=1}^{\infty} d_{m i} \zeta^{i}
$$

is holomorphic with respect to $\zeta \in \Delta$. Since $f(\mathbb{B})$ is convex,

$$
h(\zeta)=f^{-1}\left(\frac{1}{m} \sum_{k=0}^{m-1} f\left(\zeta^{1 / m} \varepsilon^{k} w\right)\right)
$$

is well-defined and holomorphic on $\Delta$. Since $f$ is normalized,

$$
f^{-1}(z)=z+O\left(|z|^{2}\right)
$$

Therefore, $h(\zeta)=d_{m} \zeta+O\left(|\zeta|^{2}\right)$. Since $h(\Delta) \subset \mathbb{B}$, we obtain $\left\|d_{m}\right\| \leq(1-\delta)^{-1}$ by applying the maximum modulus theorem with values in a complex Banach space (cf. Dunford and Schwartz [1]) to the holomorphic mapping $h(\zeta) / \zeta$ on $|\zeta|<1-\delta$. Letting $\delta$ tend to 0 , we have $\left\|d_{m}\right\| \leq 1$. Then we have

$$
\|f(\zeta w)\| \leq|\zeta|+\sum_{i=2}^{\infty}|\zeta|^{i}=\frac{|\zeta|}{1-|\zeta|}=\frac{\|\zeta w\|}{1-\|\zeta w\|}
$$

Let $D$ be a bounded convex balanced domain in $\mathbb{C}^{n}$. Then the Minkowski function of $D$ is a norm on $\mathbb{C}^{n}$ and $D$ is the unit ball with respect to the norm (cf. Jarnicki and Pflug [5]). Then the above theorem holds for $D$. In particular, the theorem gives another growth theorem of convex mappings on $D\left(p_{1}, \ldots, p_{n}\right)$ with $p_{1}, \ldots, p_{n} \geq 1$.

The author would like to thank the referee for his helpful comments and suggestions.

\section{REFERENCES}

1. N. Dunford and J. Schwartz, Linear operators, vol. 1, Interscience, New York, 1958. MR 22: 8302

2. C. H. FitzGerald and C. R. Thomas, Some bounds on convex mappings in several complex variables, Pacific J. Math. 165 (1994), 295-320. MR 95k:32021

3. S. Gong, Biholomorphic mappings in several complex variables, Contemporary Math. 142 (1993), 15-48. MR 94a:32035

4. S. Gong and T. Liu, The growth theorem of biholomorphic convex mappings on $\mathbb{B}^{p}$, Chin. Quar. Jour. Math. 6 (1991), 78-82.

5. M. Jarnicki and P. Pflug, Invariant distances and metrics in complex analysis, de Gruyter, Berlin-New York, 1993. MR 94k:32039 
6. T. Liu, The growth theorems, covering theorems and distortion theorems for biholomorphic mappings on classical domains, University of Science and Technology of China Thesis (1989).

7. T. J. Suffridge, Biholomorphic mappings of the ball onto convex domains, Abstracts of papers presented to American Mathematical Society 11(66) (1990), 46.

Faculty of Engineering, Kyushu Kyoritsu University, 1-8, Jiyugaoka, Yahatanishi-Ku, KITAKYUSHU 807, JAPAN

E-mail address: hamada@kyukyo-u.ac.jp 